\title{
Low cognitive load strengthens distractor interference while high load attenuates when cognitive load and distractor possess similar visual characteristics
}

\author{
Takehiro Minamoto • Zach Shipstead • Naoyuki Osaka • \\ Randall W. Engle
}

Published online: 27 March 2015

(C) The Psychonomic Society, Inc. 2015

\begin{abstract}
Studies on visual cognitive load have reported inconsistent effects of distractor interference when distractors have visual characteristic that are similar to the cognitive load. Some studies have shown that the cognitive load enhances distractor interference, while others reported an attenuating effect. We attribute these inconsistencies to the amount of cognitive load that a person is required to maintain. Lower amounts of cognitive load increase distractor interference by orienting attention toward visually similar distractors. Higher amounts of cognitive load attenuate distractor interference by depleting attentional resources needed to process distractors. In the present study, cognitive load consisted of faces (Experiments 1-3) or scenes (Experiment 2). Participants performed a selective attention task in which they ignored face distractors while judging a color of a target dot presented nearby, under differing amounts of load. Across these experiments distractor interference was greater in the low-load condition and smaller in the high-load condition when the content of the cognitive load had similar visual characteristic to the distractors. We also found that when a series of judgments needed to be made, the effect was apparent for the first trial
\end{abstract}

\footnotetext{
T. Minamoto $(\bowtie)$

Department of Psychology, Graduate School of Human Sciences, Osaka University, 1-2 Yamadaoka, Suita, Osaka 565-0871, Japan e-mail: txminamoto@hus.osaka-u.ac.jp

Z. Shipstead

Department of Social and Behavioral Sciences, Arizona State University, PO Box 37100, Phoenix, AZ 85069-7100, USA

N. Osaka

Department of Psychology, Faculty of Letters, Kyoto University,

Yoshida-Honmachi Sakyo, Kyoto 606-8501, Japan

R. W. Engle

School of Psychology, Georgia Institute of Technology, 654 Cherry Street NW, Atlanta, GA 30332, USA
}

but not for the second. We further tested an involvement of working memory capacity (WMC) in the load effect (Experiment 3). Interestingly, both high and low WMC groups received an equivalent effect of the cognitive load in the first distractor, suggesting these effects are fairly automatic.

Keywords Selective attention - Attentional capture . Attention and executive control · Visual working memory

Selection of relevant, rather than distracting, information requires people to maintain processing priories that bias vision toward certain information and away from other information. It is classically assumed that requiring people to maintain taskirrelevant information (i.e., cognitive load) will deplete a domain-general resource. The effect of this depletion is to make people less likely to notice critical information and more likely to be drawn to distraction as a function of the load (e.g., Lavie, Hirst, de Fockert, \& Viding, 2004). This domaingeneral assumption, however, does not always hold. In particular, we contrast two resource-specific perspectives: specialized load theory and memory-driven attention capture.

Specialized load theory (Park, Kim, \& Chun, 2007; see also Kim, Kim, \& Chun, 2005) proposes that the effect of cognitive load is resource-specific, rather than domain-general. For instance, if a person needs to make a same/different judgment on two faces while simultaneously maintaining faces in memory, performance on the judgment task will suffer due to resource competition. However, if a person needs to ignore faces while maintaining faces in memory, a same/ different judgment on other types of target information (e.g., houses) will be facilitated. This is because load is depleting 
resources that are critical to distractor processing, thus allowing efficient target selection to proceed.

Specialized load theory contrasts with the idea of memorydriven attention capture (Olivers, Meijer, \& Theeuwes, 2006; Soto, Hodsoll, Rotshtein, \& Humphreys, 2008; Soto \& Humphreys, 2007). Memory-driven attention capture predicts that attention is biased toward the type of information that is maintained in working memory. For example, Olivers et al. (2006; Experiment 2) had participants perform a visual search task after they committed a color-item to memory. Critically, the visual search contained a distractor item that was either the same or a different color as the maintained color-item. Detection of the target was slowed when the distracter's color matched the color of the maintained item, relative to when there was a mismatch.

Methodological differences aside, these studies appear to contradict each other. One reports that distractor-consistent cognitive load enhances target selection (Park et al., 2007) while the other found impairment (Olivers et al., 2006). We hypothesize that the between-study differences may be a matter of the amount of cognitive load that is being maintained. For instance, Soto and Humphreys (2008) report that impairment of selective attention decreases as cognitive load increases. On one hand, low cognitive load may act to bias available resources toward load-consistent information. High load, on the other hand, depletes resources, meaning that loadconsistent information must compete for representation in working memory. Load-consistent distractors would therefore be less likely to capture attention.

\section{The present study}

The primary goal of this study is to test the hypothesis that low specialized cognitive load impairs selective attention, while high load enhances it. To this end, we employed a task with load-consistent distractors that was based on the work of Park et al. (2007). The general procedure required participants to memorize a number of faces for later recognition. During a retention phase, participants judged the color of a dot that was superimposed on a distractor face. This is similar to a visual search task that includes a salient singleton distractor to capture attention (e.g., Theeuwes \& Burger, 1998) in a sense that a salient distractor (i.e., face) grabs attention (Theeuwes \& Van der Stigchel, 2006) and prevents participants from directing their attention toward a target.

It must be noted that the experimental task was different from the previous studies on the specialized load theory as well as the general load theory (Lavie et al., 2004; Park et al., 2007). Those studies employed Eriksen's Flanker paradigm (Eriksen \& Eriksen, 1974) for a selective attention task in which distractor influence was quantified by measuring difference in reaction time between the incongruent condition and the congruent condition. The Flanker task is highly sensitive to distractor influence under cognitive load, as it will cancel out general increases in reaction time by memory load. However, one concern is that distractor interference can be contaminated by individual differences in the ability to inhibit motor responses to incongruent distractors. More specifically, distractor interference in the incongruent condition can be underestimated for individuals with high cognitive control due to superior inhibition of prepotent behavior, while it can be overestimated in those with low cognitive control due to poor inhibition.

As the present study considers an effect of individual differences in cognitive control (i.e., working memory capacity) on distractor processing (Experiment 3), we employed a singleton-like paradigm. Similar paradigms are often used in the neuroimaging studies, which produce activation of the prefrontal cortex that seems to modulate activation of the inferior temporal lobe responding to visual distractor stimuli (Dolcos, Miller, Kragel, Jha, \& McCarthy, 2007; Jha, Fabian, \& Aguirre, 2004; Minamoto, Osaka, \& Osaka, 2010).

In Experiment 1, participants were required to maintain a prespecified number of faces in memory (no-, 1- or 3-load) while judging the color of dots that were superimposed on pictures of faces. If low specialized cognitive load biases attention toward specific visual features, then reaction times on the color judgment task should be slowest when one face is maintained. If high specialized cognitive load depletes resources for specific visual features, then reaction times should be fast when three faces must be maintained, relative to one face or no-load conditions.

In Experiment 2, the to-be-maintained stimuli were varied. Participants memorized either faces or scenes prior to performing the color judgment task with face distractors. Inclusion of the scene memory load makes it clear whether or not the effect on the distractor processing is specific to the specialized cognitive load.

In Experiment 3, we examined whether the effect of the specialized cognitive load can be attributed to individual differences in attention control ability. According to Olivers et al. (2006), memory-driven attentional capture is a relatively automatic process. If that is the case, all participants will have their attention captured by the distractors that have visual characteristics similar to items maintained in their visual working memory, regardless of individual capability of executive attentional control. However, if the executive attentional control system mediates the phenomenon, the attentional capture will be smaller for those with high attentional control relative to those with low. This was done by examining reaction times for participants who were high or low in working memory capacity (WMC), which is strongly related to several aspects of executive attention, such as prepotent response inhibition, selective attention, and novel reasoning (Conway, Cowan, \& Bunting, 2001; Kane \& Engle, 2002, 2003; 
Sobel, Gerrie, Poole, \& Kane, 2007; Unsworth, Schrock, \& Engle, 2004). Relevant to the present discussion, several neurocognitive studies that indicate people with low WMC are easily drawn to task-irrelevant information (McNab \& Klingberg, 2008; Minamoto et al., 2010; Vogel, McCollough, \& Machizawa, 2005). For example, using event-related potential, Vogel et al. (2005) demonstrated that people with low WMC specifically show contralateral activity in the posterior parietal and lateral occipital regions in response to irrelevant visual information. This was taken as evidence that these people are prone to encoding and maintaining distracting information. Comparing reaction between the high and low WMC individuals will tell us whether the attentional capture is an automatic process in nature.

\section{Sustainability of the cognitive load effect}

Although cognitive psychology and cognitive neuroscience studies have presented multiple distractors during retention period of visual working memory tasks (de Fockert, Rees, Frith, \& Lavie, 2001; Dolcos et al., 2007; Jha et al., 2004; Minamoto, Osaka, Engle, \& Osaka, 2012; Minamoto et al., 2010; Robinson, Manzi, \& Triesch, 2008), few studies have examined whether or not the effect of the memory load is stable across multiple distractors. For example, Robinson et al. (2008) required participants to remember a face or fribble (a computer generated abstract 3D object) for about 5 seconds. During the retention period, two faces were presented, and a gender-judgment task was given. The authors found that reaction time taken for the gender judgment was slower under the face load than the fribble load in both the first and second tasks, showing that the load effect is sustained for multiple presentations of distractors. The present study tested whether specialized cognitive load effects obtain across multiple distractors by including two sequentially presented color judgment tasks (with face distractors) during the retention period.

\section{Experiment 1}

Method

\section{Participants}

Forty-one graduate and undergraduate students participated in the experiment ( $M=20.44$ years, $S D=2.34,15$ females $)$. All the participants were Japanese and attended Kyoto University. Prior to an experiment, each participant provided a written informed consent (approved by the IRB of the Advanced Unit of Mind, Kyoto University). Participants were compensated with payment of 1,000 yen.

\section{Apparatus and materials}

A total of 218 Chinese face photographs were retrieved from the CAS-PEAL Face Database (Gao et al., 2008). Photographs were gray-scaled and their size were scaled to $5.1 \times 6.5 \mathrm{~cm}$, which subtended approximately $7^{\circ} \times 9^{\circ}$ of visual angle. Half of the faces were male and half the faces were female. All had neutral facial expressions. Additionally, 40 scrambled face images were prepared for the no-load condition, using a script (http://rnpsychology.org/mfiles/ ScrObjIm1.htm) running on MATLAB (MathWorks, Inc., Natick, MA). In addition to faces, two circle-stimuli filled in either red $(255,0,0)$ or green $(0,255,0)$ were prepared. Those stimuli subtended $1^{\circ} \times 1^{\circ}$ of visual angle. Stimulus presentation and response retrieval was regulated by Presentation (Neurobehavioral Systems, Inc., Albany, CA) running on Microsoft Windows 7.

\section{Procedure}

Participants were tested individually in a dark room. After providing instruction and running one practice trial, the experimenter left the room.

Figure 1 illustrates a trial sequence. Each trial began with a screen $(1,000 \mathrm{~ms})$ that informed the participant of the number of to-be-remembered faces $(0,1$, or 3 load) that would be presented (i.e., the memory load). These faces were then presented, in serial order, at the center of the screen for $2,000 \mathrm{~ms}$ with $500 \mathrm{~ms}$ fixation. In the no-load condition, a scrambled face image was presented, and participants were instructed to fixate the image. A delay of 4,000 ms followed the offset of the last face.

After this delay, the selective attention task began with the presentation of a distractor-face. After 1,000 ms a color target was added to the display for an additional 1,000 ms. The target was either red or green and presented at one of the four corners of the face distractor (top left, top right, bottom left, or bottom right). Participants were instructed to judge the color as accurately and quickly as possible by pressing a red or green key on the keyboard. Auditory feedback immediately followed the offset of the target. Two of these color judgments were performed on each trial. They were separated by a 1,000 ms interval.

Following a delay of $4,000 \mathrm{~ms}$, a face probe was presented for 3,000 ms. Participants indicated whether or not it matched an item from the memory-load set by pressing a key labeled "Y" for match or a key labeled " $N$ " for nonmatch. In the noload condition, another scrambled face was presented, and participants were instructed to press any key when they saw it. Key press was inserted in order to equate response cost in the no-load condition to the other-load conditions. Accuracy and speeded response was also emphasized for the probe task. 


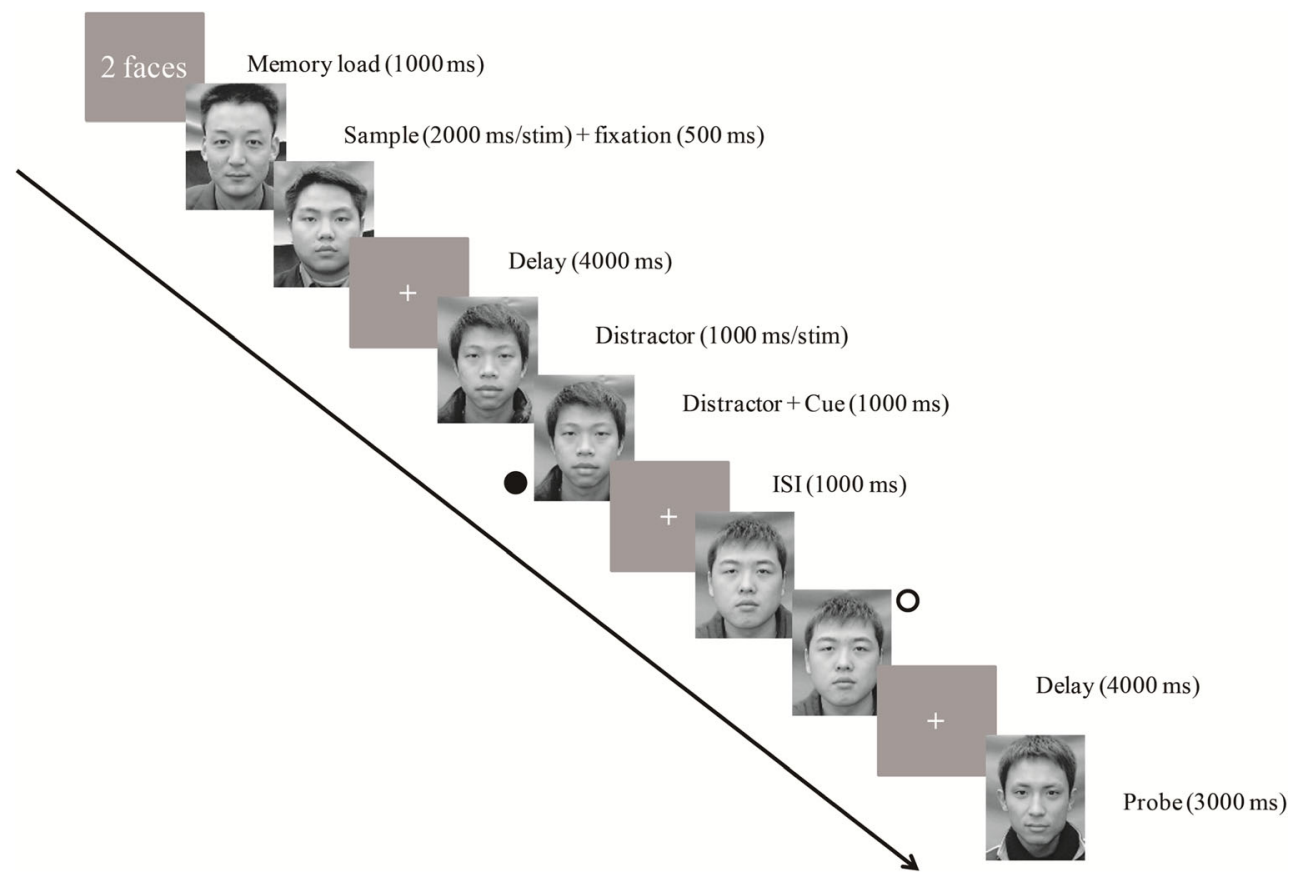

Fig. 1 A schematic diagram of the face working memory task with the selective attention task. Memory load was informed at the beginning of a trial. Sample stimuli were presented for $2,000 \mathrm{~ms}$ with a fixation $(500 \mathrm{~ms})$, which was followed by a delay of $4,000 \mathrm{~ms}$. Two selective attention tasks were performed where participants judged color of a circle stimulus (red or green) while ignoring face distractors. Each color stimulus was presented $1,000 \mathrm{~ms}$ after the onset of each face distractor. After another 4,000 ms delay, a probe stimulus was presented and participants made a decision if the probe matched the faces they had remembered
Visual feedback informed the participant of response accuracy and average performance.

Each memory-load condition consisted of 24 trials (total 72 trials). The order of these trials was randomized. Half of the trials were match trials and the other half were nonmatch. The sex of the face was consistent within a trial. Each face was presented only once during the task. As for the color stimulus, the color and the location of the stimulus were randomized across conditions. A total of six practice trials (two trials/load) were performed prior to the task. The task was self-paced. Participants were seated $41 \mathrm{~cm}$ from a 19-inch monitor. A chin rest was used to standardize and maintain the viewing distance.

\section{Design and data analysis}

A $3 \times 2$ within-subject design was used for the selective attention task, including the factors of the memory load (0-, 1-, or 3-load) and target-judgment (first vs. second). The face memory task was a one-way within-subject design, with three levels of memory load. Only correct trials were included for the statistical analysis on reaction time data. An alpha level of .05 was used as the statistical criterion. Shaffer's modified sequentially rejective Bonferroni procedure was used for multiple comparisons. All the data analysis was performed by $\mathrm{R}$ (The R Foundation for Statistical Computing).

\section{Results}

A total of eight participants were removed from the data analysis. Four participants showed poor performance in the color judgment task (2 SDs away from the group mean) even in the no-load condition, possibly due to color blindness. Another four participants showed poor performance in the memory task (2 SDs away from the group mean).

\section{Selective attention task}

Overall analysis of target judgment and memory load In the first target judgment, reaction time was slower in the 1-load condition than in the no-load and 3-load conditions (see Fig. 2). However, in the second color judgment task, reaction time was slower in the 3-load condition than the no-load condition (Fig. 2). Reaction time in the first target judgment was slower than the second one across the load.

The above statements were supported by a $2 \times 3$ ANOVA showing a significant main effect of the task order, $F(1,32)=$ $21.99, p<.001, M S E=3,682, \eta_{\mathrm{p}}^{2}=0.41$, and cognitive load, $F(2,64)=6.19, p<.01, M S E=855, \eta_{\mathrm{p}}^{2}=0.16$. An interaction was marginally significant, $F(2,64)=2.62, p=.081, M S E=$ $625, \eta_{\mathrm{p}}^{2}=0.08$.

Simple main effect analysis was performed over the marginal interaction. In the first target judgment task, a one-way 


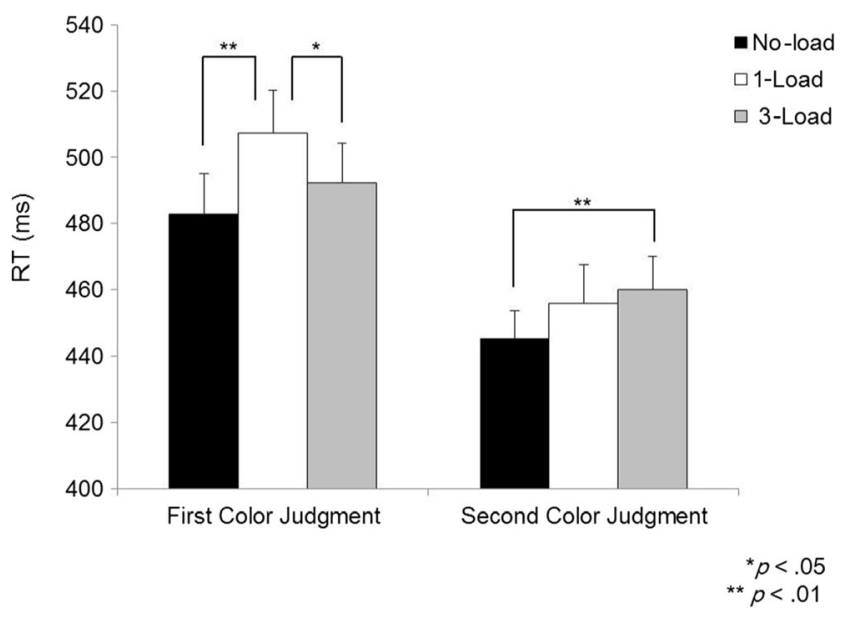

Fig. 2 Reaction time of the color judgment task in Experiment 1. In the first color judgment task, reaction time in the 1-load condition was significantly longer than that in the no-load and 3-load conditions, while reaction time did not differ between the no-load and 3-load condition. In the second color judgment task, on the other hand, response speed was slower in the 3-load condition than in the no-load condition

ANOVA showed a significant main effect of the load amount, $F(1,32)=4.56, p<.05, M S E=1,030, \eta_{\mathrm{p}}^{2}=0.13$. Shaffer's modified sequentially rejective Bonferroni procedure showed a significantly slower reaction time in the 1-load condition in comparison to the no-load condition, $t(32)=3.21, p<.01$, and the 3-load condition, $t(32)=2.19, p<.05$. Reaction time did not differ between the no-load condition and the 3-load condition, $t(32)=-1.04, p>.05$. In the second target judgment task, a main effect of the load amount was obtained, $F(1,32)=$ $4.28, p<.05, M S E=1,030, \eta_{\mathrm{p}}^{2}=0.12$. The post hoc multiple comparison showed a significantly slower reaction time in the 3-load condition than in the no-load condition, $t(32)=3.34, p<$ .01. Reaction time did not differ between the no-load condition and the 1-load condition, $t(32)=1.79, p>.05$, and the 1-load condition and the 3-load condition, $t(32)=0.82, p>.05$.

A simple main effect of the target judgment order was significant in the all load conditions: no-load, $F(1,32)=$ $19.31, p<.001, M S E=1211, \eta_{\mathrm{p}}^{2}=0.38 ; 1$-load, $F(1,32)=$ 22.76, $p<.001, M S E=1030, \eta_{\mathrm{p}}^{2}=0.42$; and 3-load, $F(1,32)$ $=9.51, p<.005, M S E=1,797, \eta_{\mathrm{p}}^{2}=0.23$.

As for the accuracy, participants showed better performance in the second target judgment than in the first one across cognitive load (see Table 1). A $2 \times 3$ ANOVA yielded a significant main effect of the order, $F(1,32)=12.68, p<.01$, $M S E=0.001, \eta_{\mathrm{p}}^{2}=0.28$, but not a main effect of the memory load, $F(2,64)=0.38, p>.05, M S E=0.001, \eta_{\mathrm{p}}^{2}=0.01$, and an interaction, $F(2,64)=0.38, p>.05, M S E=0.001, \eta_{\mathrm{p}}^{2}<.01$.

\section{Face memory task}

Memory accuracy was lower in the 3-load condition than the 1-load condition. Accuracy in simple button-press in the no-
Table 1 Mean accuracy in the color judgment task and face memory task in Experiment 1

\begin{tabular}{|c|c|c|c|c|c|c|}
\hline & \multicolumn{2}{|c|}{ No-load } & \multicolumn{2}{|l|}{ 1-load } & \multicolumn{2}{|l|}{ 3-load } \\
\hline & Mean & $\mathrm{SD}$ & Mean & SD & Mean & $\mathrm{SD}$ \\
\hline First color judgment & 0.97 & 0.05 & 0.96 & 0.04 & 0.96 & 0.04 \\
\hline Second color judgment & 0.98 & 0.03 & 0.98 & 0.04 & 0.99 & 0.02 \\
\hline Face memory & 0.98 & 0.03 & 0.97 & 0.04 & 0.91 & 0.08 \\
\hline
\end{tabular}

load condition was higher than the 1-load and 3-load conditions (see Table 1). A within-subject ANOVA showed a significant main effect of the load, $F(2,64)=23.14, p<.001$, $M S E=0.002, \eta_{\mathrm{p}}^{2}=0.42$. Post hoc multiple comparisons showed that accuracy was differed in all three comparisons: no-load vs. 1-load, $t(32)=2.28, p<.05$; no-load vs. 3-load, $t(32)=5.31, p<.001$; and 1-load vs. 3-load, $t(32)=4.81$, $p<.001$.

Reaction time in the no-load condition was the fastest among load conditions. Reaction time in the 1-load condition was faster than the 3-load condition (Fig. 3). A within-subject ANOVA showed a significant main effect of the load, $F(2$, $64)=95.35, p<.001, M S E=11,883, \eta_{p}^{2}=0.75$. Post hoc multiple comparisons showed that reaction time was significantly different in all comparisons: no-load vs. 1-load, $t(32)=-8.44, p<.001$; no-load vs. 3-load, $t(32)=-10.97, p<$ .001 ; and 1-load vs. 3-load, $t(32)=-7.91, p<.001$.

Memory Judgment

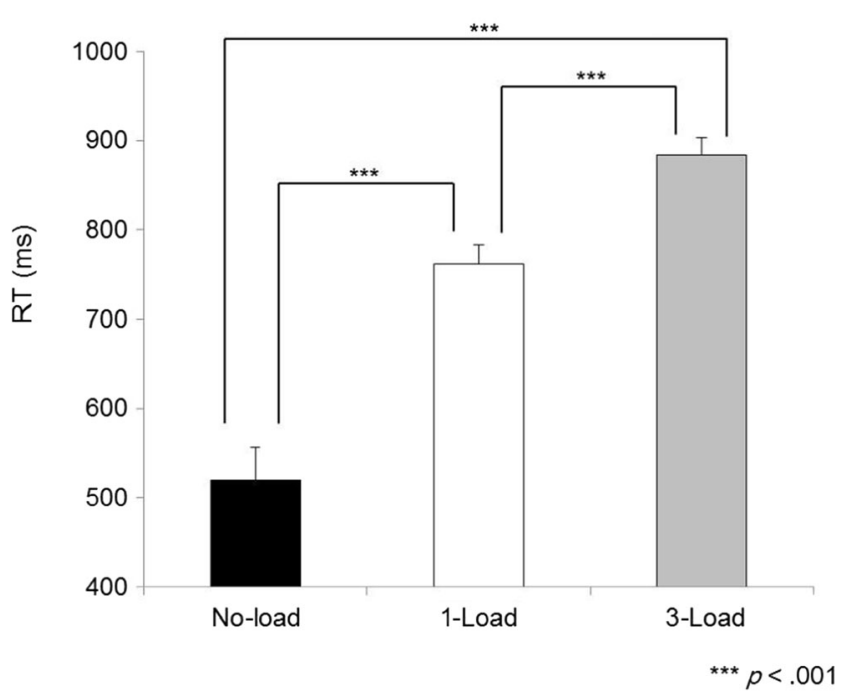

Fig. 3 Reaction time of the memory judgment in Experiment 1. Compared with the no-load condition, where participants made a button press to a scrambled image presented on a monitor, reaction time in the 1and 3-load conditions was significantly longer. Reaction time also differed between the 1-load and 3-load conditions 


\section{Discussion}

In Experiment 1, low specialized cognitive load produced greater distractor interference relative to no-load. Such interference was lowered by high specialized cognitive load; however, reaction time did not differ between the 3-load and the no-load conditions. Thus, the facilitation effect of specialized cognitive reported in the previous studies (Kim et al., 2005; Park et al., 2007) was not obtained in the current experimental paradigm. Another finding was that the effect of the specialized cognitive load was restrictive to the first selective attention task. Interestingly, in the second task, distractor interference was greater in the 3-load condition than the no-load condition, which was found in the general cognitive load studies (de Fockert et al., 2001; Lavie et al., 2004); higher cognitive load depleted cognitive resources needed to filter out irrelevant information.

\section{Experiment 2}

Experiment 1 demonstrated that distractor interference was enhanced by low specialized cognitive load but lowered by higher load. The effect, however, may not be specific to specialized cognitive load. To rule out this possibility, we included another type of visual cognitive load (scene load). Absence of attention facilitation under the high specialized cognitive load was another issue in Experiment 1. The null result may be due to absence/presence of memory load; the facilitation effect in the 3-load condition might be masked by memory load that required divided attention to perform the dual task. Therefore, we introduced the scene load to control for this possibility. In Experiment 2, participants received face and scene load in a blocked manner. Two levels of load (1- and 3-load) were administered in each visual cognitive load.

\section{Method}

\section{Participants}

Twenty-one graduate and undergraduate students at Kyoto University participated in the experiment $(M=20.95$ years, $S D=1.40,7$ females). Each participant provided a written informed consent (approved by the IRB of the Graduate School of Human Sciences, Osaka University). Participants were compensated with payment of 1,000 yen.

\section{Apparatus and materials}

Face photographs and color stimuli were identical to those used in Experiment 1. A total of 94 natural scene photographs were retrieved from Microsoft clip art (Microsoft Corporation) and IAPS (Lang, Bradley, \& Cuthbert, 2008).
All the photographs were gray scale and resized to a comparable size of faces. Ten scenes were used for a practice trial and the rest for real ones. The Presentation software regulated stimulus presentation and response recording.

\section{Procedure}

The experimental procedure was identical to Experiment 1, except for inclusion of the scene memory load. Participants performed the face load task and scene load task in different blocks, and the order of the task was counterbalanced across participants. Each task consisted of 32 trials, where half of the trials imposed one memory load and the other half did 3-load.

\section{Design and data analysis}

A $2 \times 2 \times 2$ within-subject design was used for the selective attention task, including the factors of the load type (face vs. scene), load amount (1-load vs. 3-load) and target-judgment (first vs. second). The face memory task was a $2 \times 2$ withinsubject design, which had two levels of load type (face vs. scene) and load amount (1-load vs. 3-load).

Similar to Experiment 1, accuracy and median reaction time in correct trials were analyzed, using within-subject ANOVAs. A simple effect was examined with a repeated ANOVA, when appropriate.

Results

A total of four participants were excluded from the analysis. One participant did not follow the experimental instruction, two participants showed extremely low memory performance (greater than 2 SDs from the group mean), and one participant showed extremely slow RT in the color judgment task in both the face and scene conditions (greater than 2 SDs from the group mean).

\section{Selective attention task}

Overall analysis of target judgment and memory load Consistent with Experiment 1, reaction time in the first color judgment was slower in the 1-load condition than in the 3-load condition under the face cognitive load. This difference, however, was not observed under scene load (Fig. 4, left). In addition, reaction time in the 1-load condition was slower under face load than scene load, while those in the 3load condition did not differ between the face and scene conditions (Fig. 4, left).

Also consistent with Experiment 1, reaction time in the second color judgment task was shorter than that in the first color judgment. Additionally, reaction time was slower under the face load than the scene load across load amount (Fig. 4, 
First Color Judgment

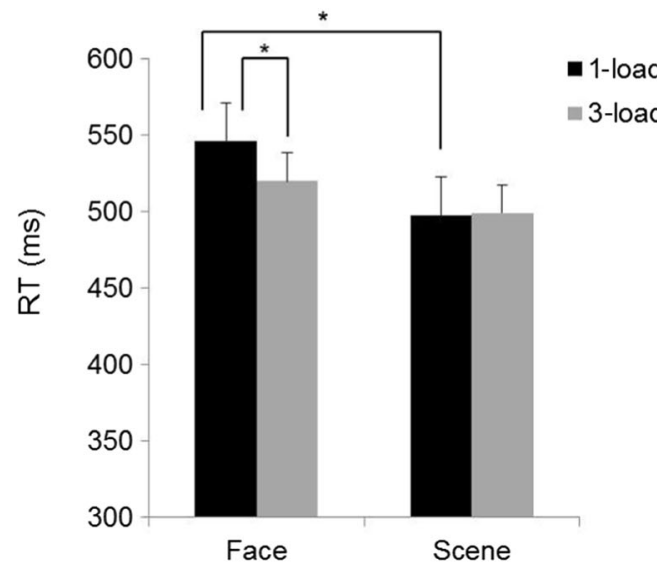

Fig. 4 Reaction time of the first color judgment task and the second color judgment task shown by the load type in Experiment 2. In the first color judgment task (left), reaction time in the 1-face-load condition was slower than that in the 3-face-load condition. Additionally, reaction time in the 1-

right), and reaction time was slower under 3-load than 1-load across the type of load (Fig. 5 left).

A $2 \times 2 \times 2$ ANOVA showed a main effect of load type, $F(1,16)=5.06, p<.05, M S E=8077, \eta_{\mathrm{p}}^{2}=0.24$, and that of target judgment order, $F(1,16)=22.57, p<.001, M S E=$ $5277, \eta_{\mathrm{p}}^{2}=0.59$. A significant interaction was found between the factors of load amount and the target judgment order, $F(1$, 16) $=13.29, p<.005, M S E=440, \eta_{\mathrm{p}}^{2}=0.45$. A three-way interaction between the three factors was marginally significant, $F(1,16)=3.33, p=.087, M S E=636, \eta_{\mathrm{p}}^{2}=0.17$. Other main effects and interactions were not significant: a main effect of load amount, $F(1,16)=0.05, p>.05, M S E=474, \eta_{\mathrm{p}}^{2}<$ .01 ; an interaction between load type and load amount, $F(1$,

\section{Second Color Judgment}

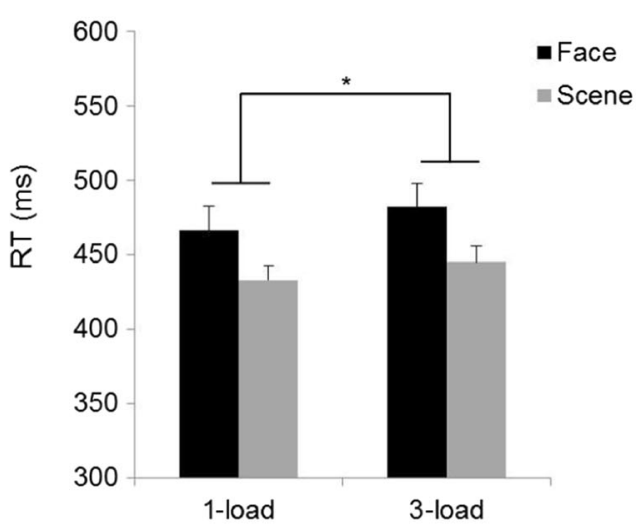

Fig. 5 Reaction time in the second color judgment task shown by the load amount (left) and that the memory recognition (right) in Experiment 2. In the second color judgment task, reaction time in the 3-load condition
Second Color Judgment

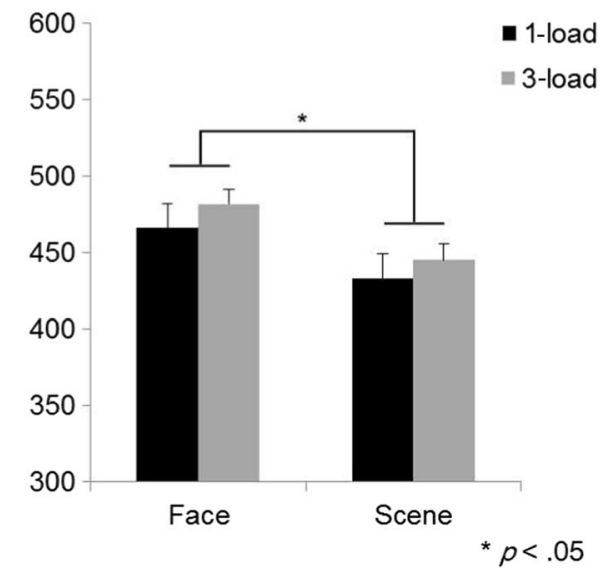

face-load condition was slower than that in the 1-scene load condition, while reaction time did not differ between the 3-face-load and the 3scene-load conditions. In the second color judgment task (right), reaction time in the face conditions was longer than those in the scene conditions

16) $=2.38, p>.05, M S E=506, \eta_{\mathrm{p}}^{2}=0.13$; an interaction between load type and target judgment order, $F(1,16)<.01$, $p>.05, M S E=2179, \eta_{\mathrm{p}}^{2}<.01$.

\section{Analysis of load type}

Since the three-way interaction trended toward significance, we analyzed the reaction time data in the first and second target judgment task separately. In the first target judgment, a $2 \times 2$ ANOVA showed a significant main effect of the load amount, $F(1,16)=5.21, p<.05, M S E=493, \eta_{\mathrm{p}}^{2}=0.26$, and a significant trend of interaction between the factors of load type and load amount, $F(1,16)=4.31, p=.054, M S E=757$,

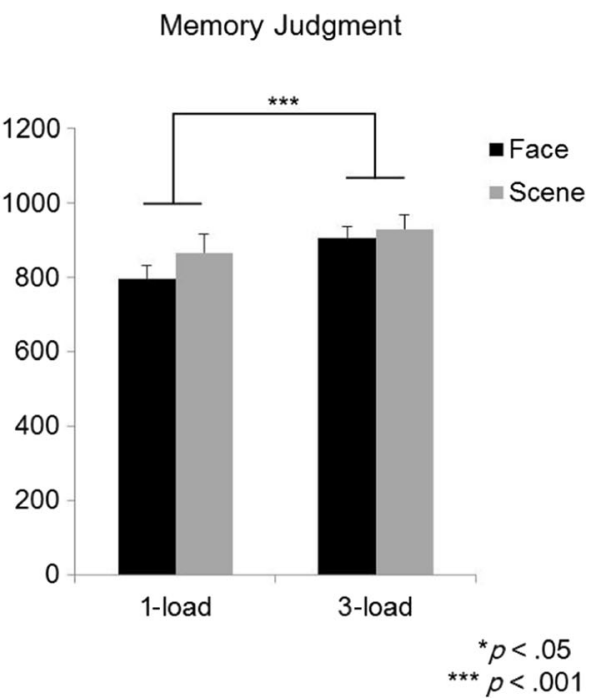

was slower than those in the 1-load condition across the load type. Similarly, reaction time of the memory recognition was slower in the 3-laod condition than the 1-load condition across the load type 
$\eta_{\mathrm{p}}^{2}=0.21$, but not a main effect of load type, $F(1,16)=2.67$, $p>.05, M S E=7,600, \eta_{\mathrm{p}}^{2}=0.14$. The post hoc analysis on simple effects for the interaction showed a significant effect of load amount under the face load, $F(1,16)=7.15, p<.05$, MSE $=814, \eta_{\mathrm{p}}^{2}=0.31$. The 1 -face-load produced significantly longer reaction time than the 3 -face-load. In addition, the simple effect analysis showed a significant effect of the load type under the 1-load, $F(1,16)=4.92, p<.05, M S E=4,001$, $\eta_{\mathrm{p}}^{2}=0.24$. That is, the 1 -face-load produced a significantly longer reaction time than the 1 -scene-load. Other effects did not reach the significant level $(p>.05)$.

In the second target judgment, a $2 \times 2$ ANOVA showed a significant main effects of load type, $F(1,16)=7.77, p<.05$, $M S E=2,656, \eta_{\mathrm{p}}^{2}=0.33$, and load amount, $F(1,16)=7.84$, $p<.05, M S E=420, \eta_{\mathrm{p}}^{2}=0.33$, but not an interaction between the factors, $F(1,16)=0.69, p>.05, M S E=385, \eta_{\mathrm{p}}^{2}=0.01$.

As for the accuracy, all the participants showed perfect performance in the 3 -scene-load condition in the second target judgment (see Table 2). Therefore, we only analyzed the first color judgment data. The first target judgment in the face condition was less accurate than that in the scene condition across the cognitive load. A $2 \times 2$ ANOVA showed a main effect of load type, $F(1,16)=5.49, p<.05, M S E=0.002$, $\left.\eta_{\mathrm{p}}^{2}=0.25\right]$; but not of load amount, $F(1,16)=0.03, p>.05$, $M S E=0.026, \eta_{\mathrm{p}}^{2}<.01$; or an interaction, $F(1,16)=0.69$, $p>.05, M S E=0.002, \eta_{\mathrm{p}}^{2}=0.04$.

\section{Memory task}

Memory retrieval took longer time in the 3-load condition than in the 1-load condition across the face and scene cognitive load (Fig. 5, right). As for the accuracy, participants showed poorer performance in the face load condition than the scene load condition across load amount, in addition to the lower performance in the 3-load condition than the 1-load condition (Table 2).

A $2 \times 2$ ANOVA involving reaction time showed a main effect of load amount, $F(1,16)=26.37, p<.001, M S E=17$, $803, \eta_{\mathrm{p}}^{2}=0.62$; but not a main effect of load type, $F(1,16)=$ $2.02, p>.05, M S E=284,851, \eta_{\mathrm{p}}^{2}=0.11$; or an interaction, $F(1,16)=1.22, p>.05, M S E=7462, \eta_{\mathrm{p}}^{2}=0.07$.
A $2 \times 2$ ANOVA involving accuracy yielded significant main effects of load type, $F(1,16)=11.74, p<.005, M S E=$ $0.003, \eta_{\mathrm{p}}^{2}=0.42$, and load amount, $F(1,16)=13.74, p<.005$, $M S E=0.003, \eta_{\mathrm{p}}^{2}=0.46$; but not an interaction, $F(1,16)=$ $0.01, p>.05, M S E=0.003, \eta_{\mathrm{p}}^{2}<0.01$.

\section{Discussion}

In the second experiment, interference by face distractors was observed under low face cognitive load but not under low scene load. The interference did not differ between the high face load condition and the high scene load condition. These results support the hypothesis that low specialized cognitive load produced greater category-congruent distractor interference while high load lowered it. Once again the effect was found only in the first selective attention task, but not in the second. In the second task, distractor interference was greater in the face load conditions than in the scene load conditions, regardless of load amount. It was also found that distractor interference was greater under high load than under low load, regardless of the load type, which is somewhat consistent with Experiment 1 in a sense that higher cognitive load increased distractor interference (although reaction time did not differ between the 1-load condition and 3-load condition in Experiment 1). Regarding the facilitation effect of the specialized cognitive load, we failed to obtain the effect when comparing reaction time in the 3 -face-load condition with that in the 3 -scene load condition.

\section{Experiment 3}

Two experiments have shown that distractor interference was greater under low cognitive load and smaller under high cognitive load. Such load effects were obtained in the first target judgment but not in the second. The second experiment further refined our understanding of this effect by demonstrating that it is specific to load that contains characteristics that are visually similar to the distractor. The third experiment explores whether executive attentional control could mediate

Table 2 Mean accuracy in the color judgment task and face/scene memory tasks in Experiment 2

\begin{tabular}{|c|c|c|c|c|c|c|c|c|}
\hline & \multicolumn{4}{|l|}{ Face } & \multicolumn{4}{|l|}{ Scene } \\
\hline & \multicolumn{2}{|l|}{ 1-load } & \multicolumn{2}{|l|}{ 3-load } & \multicolumn{2}{|l|}{ 1-load } & \multicolumn{2}{|l|}{ 3-load } \\
\hline & MEAN & SD & MEAN & SD & MEAN & SD & MEAN & SD \\
\hline First color judgment & 0.94 & 0.07 & 0.96 & 0.06 & 0.98 & 0.05 & 0.97 & 0.05 \\
\hline Second color judgment & 0.99 & 0.03 & 0.98 & 0.04 & 0.98 & 0.03 & 1.00 & 0.00 \\
\hline Memory & 0.94 & 0.08 & 0.89 & 0.06 & 0.99 & 0.03 & 0.94 & 0.05 \\
\hline
\end{tabular}


the effect of the cognitive load, employing an individual differences approach.

\section{Methods}

\section{Participants}

Twenty high and 20 low WMC participants (aged 18 to 35 years) were selected from a database of prescreened community members and Georgia Tech students $(N=264)$. WMC was measured via several complex span tasks (Daneman \& Carpenter, 1980; Turner \& Engle, 1989; Unsworth, Heitz, Schrock, \& Engle, 2005) in the present study. Following the procedure of Unsworth and Engle (2007), screening tests included operation span, reading span, and symmetry span (described below). Participants from the top quartile were categorized as high in WMC (high span) while participants from the bottom quartile were categorized as in low WMC (low span). All the participants provided written consent (approved by Georgia Tech IRB) before beginning the experiment. Participants were compensated with either course credit or payment of 20 dollars.

\section{Prescreening tasks}

Operation span All three screening tasks were complex span tasks in which participants are required to remember a list of items, the presentation of each is interrupted by the requirement to complete a processing task. In the automated operation span task (Unsworth et al., 2005), participants first mentally solved a simple mathematical equation after which a single letter was presented for $1,000 \mathrm{~ms}$. This process repeated until three to seven unrelated letters had been displayed $(\mathrm{F}, \mathrm{H}$, J, K, L, N, P, Q, R, S, T, Y). Participants then attempted to recreate the sequence of letters by clicking a response screen that was presented via computer monitor. Each possible list length occurred three times, in random order. Test score were computed by adding the number of items correctly recalled their original position. The same scoring procedure was also applied to reading span test and symmetry span test.

Reading span The automated reading span (Unsworth, Redick, Heitz, Broadway, \& Engle, 2009) was similar to the operation span, with the exception that rather than solving a mathematical equation, participants decided whether a presented sentence was semantically appropriate. Half of the sentences made sense while the other half did not.

Symmetry span The automated symmetry span task (Unsworth et al., 2009) required participants to judge whether or not a figure was symmetrical about its vertical axis, after which they were shown a $4 \times 4$ grid in which one to-beremembered space was colored red $(650 \mathrm{~ms})$. The figure used in the symmetry judgment task consisted of an $8 \times 8$ matrix in which a certain number of squares had been colored black. Figures were symmetrical in half of the trials and asymmetri$\mathrm{cal}$ in the rest of the trials. Participants repeated this procedure two to five times, and then were required to recall the spaces that had been highlighted in the order that they had been highlighted by clicking locations in an empty $4 \times 4$ matrix. Each possible list length was used three times, in random order.

\section{Apparatus and materials}

Face photographs and color stimuli were identical to Experiments 1 and 2. It should be noted that Chinese faces minimized the potential for same-race effects (Horry, Wright, \& Tredoux, 2010; Malpass \& Kravitz, 1969), as our sample was primarily composed of white/Caucasians and black/ African Americans. ${ }^{1}$ Stimulus presentation and response retrieval was regulated by E-Prime 2.0.8.22 (Psychology Software Tools, Inc., Sharpsburg, PA) running on Microsoft Windows Vista.

\section{Procedure}

The experiment procedure was almost identical to the above experiments. Participants performed three levels of face load (1-, 2-, and 3-load). We included the 2-load condition because of the documented difficulty of remembering faces of other races (Horry et al., 2010; Malpass \& Kravitz, 1969). Each memory load condition consisted of 20 trials (total 60 trials). The order of these trials was randomized.

\section{Design}

For the selective attention task, the design was a $2 \times 3 \times 2$ mixed model, with WMC (high vs. low) as between-subject factor and memory load (1-, 2- and 3-load) and targetjudgment (first vs. second) as within-subject factors. The face memory task was a $2 \times 3$ factorial design, with two levels of WMC and three levels of memory load.

\section{Data analysis}

In the selective attention task, mean accuracy in the color judgment and median reaction time for correct responses were analyzed, using a mixed ANOVA. Likewise, in the face working memory task, mean accuracy and median reaction time at correct recognition was analyzed with the mixed ANOVA.

\footnotetext{
${ }^{1}$ One high WMC participant was of East Asian decent. The exclusion of this participant did not change the nature of our analysis, so we retained his data.
} 


\section{Results}

Four participants were excluded from the analysis because two could not complete the task and the other two showed lower accuracy on the selective attention task (2 SDs away from the mean). All were individuals from the low WMC group. Therefore, a total of 36 participants were included in the analysis (20 high spans and 16 low spans). The mean $z$ score of the high span participants was $0.98(S D=0.24)$ and that of the low span participants was $-1.08(S D=0.41)$. The mean ages for the high span participants was $21.45(S D=$ $3.30)$ and that of the low span participants was $24.41(S D=$ $3.61)$, respectively.

\section{Selective attention task (all participants)}

Consistent with the two experiments above, participants responded slowly in making the first target judgment relative to the second one. More importantly, the effect of the cognitive load was restricted to the first target judgment. That is, participants showed slower response in the 1-load condition than the other two conditions in the first-target judgment but not in the second (see Fig. 6).

A $2 \times 3 \times 2$ ANOVA over reaction time showed a significant main effect of load amount, $F(2,68)=5.57, p<.01$, $M S E=1,668, \eta_{\mathrm{p}}^{2}=0.14$, and target-judgment order, $F(1,34)=15.82, p<.001, M S E=7044, \eta_{p}^{2}=0.31$. A significant interaction was found between load amount and target-judgment order, $F(2,68)=4.02, p<.05, M S E=$ $1339, \eta_{\mathrm{p}}^{2}=0.10$. The main effect of WMC was marginal, $F(1,34)=3.64, p=.064, M S E=23,353, \eta_{\mathrm{p}}^{2}=0.10$. Interactions were not significant between WMC and load amount, $F(2,68)=0.133 p>.05, M S E=1,668, \eta_{\mathrm{p}}^{2}<0.01$, or between the factor of WMC and the target-judgment order,

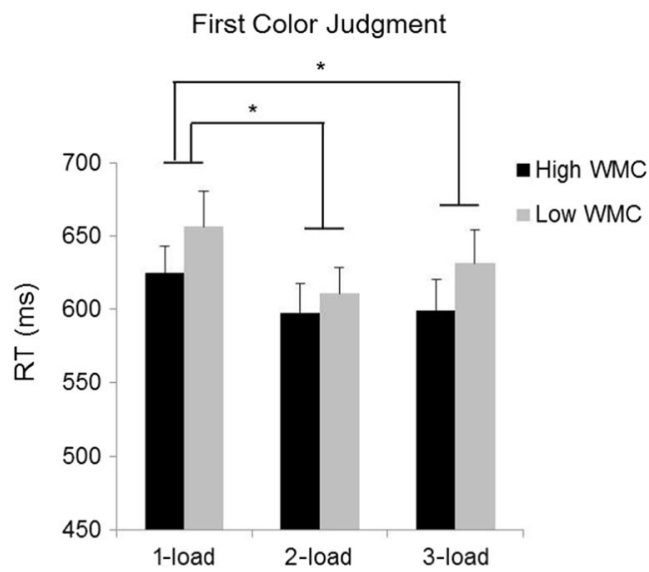

Fig. 6 Reaction time of the color judgment task in Experiment 3. In the first color judgment task (left), both high and low working memory capacity (WMC) individuals showed slower reaction time in the 1-load condition than in the 2- and 3-load conditions. On the other hand, in the
$\left.F(1,34)=1.43, p>.05, M S E=7,044, \eta^{2}=0.03\right]$. An interaction of the three factors was not significant, either, $F(2,68)=0.81, p>.05, M S E=1,339, \eta^{2}=0.03$.

Simple main effect analyses were performed over the load $\mathrm{x}$ judgment-order interaction. As the result, a significant main effect of load amount was obtained in the first target judgment, $F(2,68)=6.77, p<.005, M S E=1,853, \eta_{\mathrm{p}}^{2}=0.17$, but not in the second judgment, $F(2,68)=1.84, p>.05, M S E=1,154$, $\eta_{\mathrm{p}}^{2}=0.05$. Shaffer's modified sequentially rejective Bonferroni procedure test showed a slower reaction time in the 1-load condition than in the 2-load, $t(34)=3.12, p<.05$, and 3-load condition, $t(34)=2.45, p<.05$. Reaction time did not differ between the 2-load and 3-load condition, $t(34)=$ $1.38, p>.05$. Simple main effects of the judgment order were significant in the 1-load, $F(1,34)=19.48, p<.001, M S E=3$, $363, \eta_{\mathrm{p}}^{2}=0.36$, and 3-load conditions, $F(1,34)=14.69, p<$ $.001, M S E=3,000, \eta_{\mathrm{p}}^{2}=0.30$, and significant trend in the 2load condition, $F(1,34)=3.75, p=.061, M S E=3,358$, $\eta_{\mathrm{p}}^{2}=0.10$.

Accuracy on the target-judgment task showed a similar pattern to the reaction time. In the first target judgment, accuracy was lower in the 1-load condition than in the 2- and 3load conditions. On the other hand, such difference was no longer available in the second judgment (see Table 3). Another similarity was that accuracy was lower in the first target judgment than in the second one.

A $2 \times 3 \times 2$ ANOVA showed a significant main effect of judgment order, $F(1,34)=17.77, p<.001, M S E=0.007$, $\eta_{\mathrm{p}}^{2}=0.34$, and an interaction between load amount and judgment order, $F(2,68)=7.07, p<.005, M S E=0.003, \eta_{\mathrm{p}}^{2}=$ 0.17 . Main effects were not significant for WMC, $F(1,34)=$ $0.17, p>.05, M S E=0.017, \eta_{\mathrm{p}}^{2}<0.01$, or load amount, $F(1,34)=1.14, p>.05, M S E=0.004, \eta_{\mathrm{p}}^{2}=0.03$. Other two-way interactions were insignificant: WMC x load

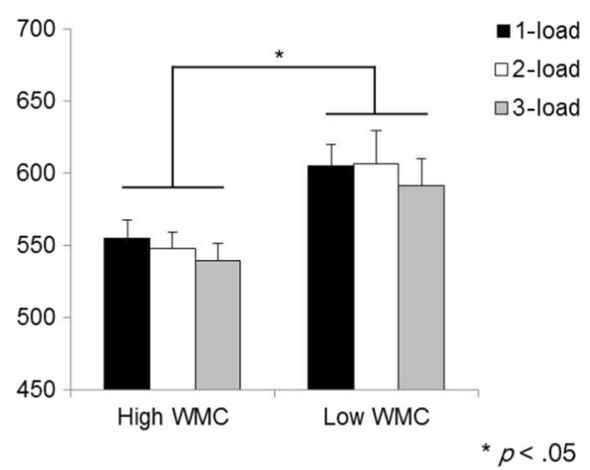

second color judgment task (right), the load effect was no longer observed while the factor of the WMC was obtained; reaction time in the high WMC group was faster than the low WCM groups across load 
Table 3 Mean accuracy in the color judgment task and face memory task in Experiment 3

\begin{tabular}{|c|c|c|c|c|c|c|c|}
\hline & \multirow[b]{2}{*}{ WMC } & \multicolumn{2}{|l|}{ 1-load } & \multicolumn{2}{|l|}{ 2-load } & \multicolumn{2}{|l|}{ 3-load } \\
\hline & & Mean & SD & Mean & SD & Mean & SD \\
\hline \multirow[t]{2}{*}{ First color judgment } & High & 0.88 & 0.08 & 0.91 & 0.12 & 0.91 & 0.08 \\
\hline & Low & 0.87 & 0.11 & 0.93 & 0.08 & 0.90 & 0.08 \\
\hline \multirow{2}{*}{$\begin{array}{l}\text { Second color } \\
\text { judgment }\end{array}$} & High & 0.96 & 0.05 & 0.96 & 0.06 & 0.95 & 0.06 \\
\hline & Low & 0.96 & 0.06 & 0.93 & 0.07 & 0.95 & 0.06 \\
\hline \multirow[t]{2}{*}{ Face memory } & High & 0.95 & 0.05 & 0.90 & 0.09 & 0.90 & 0.08 \\
\hline & Low & 0.88 & 0.08 & 0.88 & 0.11 & 0.86 & 0.10 \\
\hline
\end{tabular}

amount, $F(2,68)=0.01, p>.05, M S E=0.004, \eta_{\mathrm{p}}^{2}<0.01$, WMC x judgment order, $F(1,34)=0.35, p>.05, M S E=$ $0.007, \eta_{\mathrm{p}}^{2}<.01$. A three-way interaction was not significant, either, $F(2,68)=1.50, p>.05, M S E=0.003, \eta_{p}^{2}=0.04$.

Simple main effect analyses examining load x judgmentorder interaction yielded a significant main effect of the load amount in the first target-judgment task, $F(2,68)=4.14, p<$ $.05, M S E=0.005, \eta_{\mathrm{p}}^{2}=0.11$, but not in the second, $F(2,68)=$ $1.26, p>.05, M S E=0.002, \eta_{\mathrm{p}}^{2}=0.04$. Post hoc multiple comparison tests showed a lower accuracy in the 1-load condition than in the 2-load, $t(34)=2.79, p<.05$, and 3-load conditions, $t(34)=2.02, p=.051$. Accuracy did not differ between the 2-load and 3-load condition, $t(34)=1.38, p>.05$.

Because the cognitive load affected the first targetjudgment but not the second, we analyzed the performance of first and the second target-judgment separately to examine the effect of the working memory capacity.

\section{Selective attention task (by WMC)}

In the first target judgment, high and low WMC groups did not differ and had slower reaction time in the 1-load condition than in the other conditions (Fig. 6, left). Similarly, accuracy did not differ between groups.

A $2 \times 3$ ANOVA over the reaction time data showed a significant main effect of load amount, $F(2,68)=6.77, p<$ $.005, M S E=1,853, \eta_{\mathrm{p}}^{2}=0.17$, but not a main effect of WMC, $F(1,34)=0.92, p>.05, M S E=19,938, \eta_{\mathrm{p}}^{2}=0.03$, or an interaction, $F(2,68)=0.61, p>.05, M S E=1,853, \eta_{\mathrm{p}}^{2}=0.01$. The results of the multiple comparisons were described above.

Similarly, a $2 \times 3$ ANOVA over the accuracy data showed a significant main effect of load amount, $F(2,68)=4.14, p<$ $.05, M S E=0.005, \eta_{\mathrm{p}}^{2}=0.11$, but not a main effect of $\mathrm{WMC}$, $F(1,34)<.01, p>.05, M S E=0.017, \eta_{\mathrm{p}}^{2}<.01$, or an interaction, $F(2,68)=0.46, p>.05, M S E=0.005, \eta_{\mathrm{p}}^{2}=0.01$.

In the second target-judgment task, on the other hand, high WMC group showed a shorter reaction time than the low WMC group across the load amount (Fig. 6, right), while accuracy did not differ between the groups.
A $2 \times 3$ ANOVA over the reaction time data showed a significant main effect of WMC, $F(1,34)=7.35, p<.05$, $M S E=10,460, \eta_{\mathrm{p}}^{2}=0.18$, but not a main effect of load amount, $F(2,68)=1.84, p>.05, M S E=1,154$, $\eta_{\mathrm{p}}^{2}=0.05$, or an interaction, $F(2,68)=0.15, p>.05$, $M S E=1,154, \eta_{\mathrm{p}}^{2}<0.01$.

A $2 \times 3$ ANOVA over the accuracy data did not show significant main effects of WMC, $F(1,34)=0.77, p>.05$, $M S E=10.007, \eta_{\mathrm{p}}^{2}=0.02$, load amount, $F(2,68)=1.26, p>$ $.05, M S E=0.001, \eta_{\mathrm{p}}^{2}=0.03$, or an interaction, $F(2,68)=$ $0.86, p>.05, M S E=0.001, \eta_{\mathrm{p}}^{2}=0.02$.

\section{Face memory task}

On the whole, participants performed better in the 1-load condition than in the 3-load condition (see Table 3). As expected, individuals with high WMC showed better memory performance than those with low WMC (see Table 3). However, load and WMC did not interact.

A mixed ANOVA on accuracy yielded a significant main effect of memory load, $F(2,68)=3.25, p<.05, M S E=0.004$, $\eta_{\mathrm{p}}^{2}=0.09$; and WMC, $F(1,34)=4.15, p<.05, M S E=0.01$, $\eta_{\mathrm{p}}^{2}=0.11$; but not an interaction, $F(2,68)=2.09, p>.05$, $M S E=0.004, \eta_{\mathrm{p}}^{2}=0.06$. The post hoc multiple comparisons showed a lower accuracy in the 3-load condition than in the 1load condition, $t(34)=2.76, p<.05$. Accuracy did not differ between the 1-load and 2-load conditions, $t(34)=1.68, p>$ .05 , and the 2-load and 3-load conditions, $t(34)=0.42, p>.05$.

As for the reaction time, participants responded faster in the 1-load condition in comparison to the 2 - and 3-load conditions (see Fig. 7). A $2 \times 3$ ANOVA showed a significant main effect of the memory load, $F(2,68)=21.19, p<.001, M S E=5,917$, $\eta_{\mathrm{p}}^{2}=0.38$, but not a main effect of WMC; $F(1,34)=2.82, p>$ $.05, \mathrm{MSE}=82,679, \eta_{\mathrm{p}}^{2}=0.08$; and an interaction, $F(2,68)=$ $2.18, p>.05, M S E=5,917, \eta_{p}^{2}=0.06$. The multiple

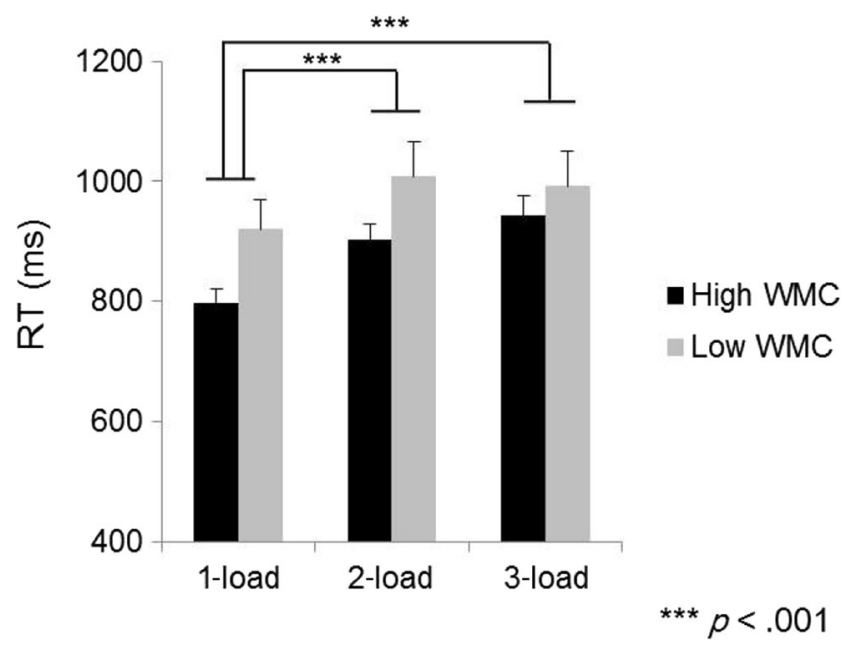

Fig. 7 Reaction time of the memory task in Experiment 3. Reaction time in the 1-load condition was significantly shorter than in the 2- and 3-load conditions 
comparison tests revealed significant differences in reaction time between the 1-load condition and the 2-load, $t(34)=6.25$, $p<.001$, and 3-load conditions, $t(34)=5.66, p<.001$. Reaction time did not differ between the 2-load and 3-load conditions, $t(34)=0.65, p>.05$.

It should be noted that accuracy as well as reaction time did not differ between the 2-load and 3-load condition. The result might be obtained due to a capacity limitation of Chinese face stimuli for non-Asian participants. Considering the finding that complexity of an object determines the capacity limit of visual working memory (Alvarez \& Cavanagh, 2004) and memory for other-race is poorer than that for self-race (Horry et al., 2010; Malpass \& Kravitz, 1969), it is reasonable to assume that capacity limit of Chinese face information was around two for the participants.

\section{Discussion}

Consistent with Experiments 1 and 2, distractor interference was greater under the low-load condition than the high-load conditions. Again, the effect was restricted to the first selective attention task. Importantly, the effect did not differ between the high and low WMC groups, indicating that the effect of the specialized cognitive load on category-congruent distractor interference may not be modulated by executive attentional control. In the second selective attention task, high cognitive load did not produce greater distractor interference found in Experiments 1 and 2. However, we found an effect of the WMC, where the low WMC group showed greater interference than the high WMC group. The result may indicate that executive attentional control mediates selective attention only after the specialized cognitive load effect on distractor interference disappears. However, the result must be carefully interpreted, as a three-way interaction was not significant.

\section{General discussion}

Specialized cognitive load and selective attention

The present study examined how selective attention was affected by specialized cognitive load that had overlapping features with the to-be-ignored distractors. Manipulating the amount of cognitive load, we showed that target selection for the first color judgment task was slower in the low specialized cognitive load (1-load) condition than the high specialized cognitive load conditions across the three experiments. Experiment 1 provided evidence that low specialized cognitive load produced greater distractor interference relative to no cognitive load, while high specialized cognitive load attenuated the interference to the level of the no-load condition. Using the scene cognitive load, Experiment 2 showed that such an effect was obtained only when visual characteristics of the distractor stimuli were similar to that of the cognitive load. Additionally, the results of Experiment 3 suggest that memory-driven attentional capture (enhanced distractor interference under the low specialized cognitive load) is an automatic process, since both high and low WMC groups showed a greater interference in the low-load condition relative to the high-load conditions.

Distractor interference was greatest when specialized cognitive load was low, which is consistent with the previous studies proposing memory-driven attentional capture. Attentional capture is thought to emerge through an attentional bias produced by a specific representation held in working memory (Olivers et al., 2006; Soto et al., 2008; Soto \& Humphreys, 2007). Conversely, when specialized cognitive load was high, such attentional capture was no longer observed; reaction time for the first color judgment did not differ between the high load and no-load condition. We interpret this as indicating that high cognitive load fully consumed attentional resources specially assigned for faces, which did not allow distractor faces to be processed. It is the amount of specialized cognitive load that determines whether memorydriven attentional capture emerges. On one hand, if the cognitive load is low and specialized attentional resource remains, selective attention is biased toward items that have similar visual characteristics. On the other hand, if the specialized cognitive load is high, characteristic-specific attentional resources are fully consumed and such attentional bias is no longer obtained.

In the high-load condition, enhancement of selective attention was not observed relative to the no-load condition. On its face, this is inconsistent with the findings of Park et al. (2007). However, methodological differences of measuring distractor interference must be considered. As discussed in the introduction, Park et al. employed the Flanker task in their selective attention task. In that study, distractor interference was computed by subtracting reaction time in the congruent condition from that in the incongruent condition. Under specialized cognitive load (e.g., face), effects from face distractors were eliminated. Both facilitation effects in the congruent condition and interference effects in the incongruent condition were removed, producing small differences in reaction time, compared with no-load condition.

In the present study, we measured raw reaction time for the color judgment task preceded by a face distractor, and defined it as the index of distractor interference. Therefore, memory load was not cancelled out, which could prevent the facilitation effect which was found in the study of Park et al. (2007). In fact, reaction time itself was slower under specialized cognitive load than no-load condition in Park et al. In Experiment 2, we introduced scene load and compared magnitude of the distractor interference between the 3-face load condition and 3 -scene load condition. Again, facilitation effect was not obtained under the high face load, even though scene memory 
load was given. However, accuracy in the 3-face load condition was the lowest among four conditions, which could have prevented a facilitation effect. Thus, further investigation is required to examine the facilitation effect by high specialized cognitive load within the current experimental paradigm. It should be noted, however, that the high specialized cognitive load lowered the interference effect across all three experiments relative to the low load.

Finally, it remains unclear which property of a distractor produced lower distractor interference. Although we argue that visual similarity plays a significant role, it is hard to exclude the possibility that visual category contributed the attenuated effect in the present study. However, Cheung and Gauthier (2010) showed that a child face load interfered with holistic processing of a subsequent child face but not that of an adult face. In their task, a face picture was divided into two parts (top and bottom), and participants were instructed to remember the top part of the face. Critically, two conditions were prepared (congruent and incongruent). In the congruent condition, the bottom part of a probe stimulus was identical to the memory stimulus, while in the incongruent condition, it was different from the memory item. If holistic processing of the face item occurred, memory performance in the former condition should be increased (congruency effect) while that in the latter condition should be decreased (incongruency effect). Next, they tested an effect of the face load (child vs. adult) on the child or adult face composite task. The result showed that the congruency and incongruency effects were observed only when type of the face load and that of the face composite task was different. Based on the finding the authors concluded that "interference in visual working memory is produced by the limited capacity for visually or psychologically similar representations (pp. 460)." The finding favors the present conclusion that change in distractor interference by the specialized cognitive load depends on visual similarity between a load and distractor item, rather than visual category. However, further study, which parametrically manipulates visual similarities between load of cognitive load and distractor stimulus, is mandatory in order to exclude the possibility stated above.

\section{Memory-driven attentional capture as an automatic cognitive process}

Soto et al. (2008) proposed that memory-driven attentional capture is an automatic process, and executive attentional control has little involvement. In order to test the hypothesis, we compared reaction time for the color judgment task between the high and low WMC individuals in Experiment 3. Both high and low WMC group showed slower reaction time in the low-load condition than the high-load condition for the critical first judgment. This result indicates that when cognitive load is low, both WMC groups bias their attention toward distractor items that have characteristics that are similar to the contents of visual working memory. Given that WMC reflects individual efficiency in attentional control (Conway et al., 2001; Kane \& Engle, 2002, 2003; Sobel et al., 2007; Unsworth et al., 2004), a high WMC group should have superior resolution of distractor interference to a low WMC if this bias were affected by controlled processes. However, low cognitive load impaired target selection in both high and low WMC in a similar fashion, suggesting that executive attentional control does not mediate memory-driven attentional capture; that is, the attentional capture is a fairly automatic process.

\section{Sustainability of the effect of the specialized cognitive load}

The bidirectional effect of the specialized cognitive load was only obtained in the first target judgment task. The effect was no longer observed in the second task. This pattern was consistent across three experiments, indicating that the effect of the specialized cognitive load on distractor interference is apparent for the first distractor but vanishes after visually similar distractors are encountered. The results were inconsistent with the finding in Robinson et al. (2008), where effect of the face working memory load delayed reaction time for both the first and second gender judgment tasks inserted in a delay period. The critical difference between the previous study and the present study is that the former required active processing of distractor faces (relative to the target memoranda) for gender judgments while the latter required active filtering of those faces. Thus, it seems that, in situations where a visuallysimilar distractor is task-irrelevant and should be ignored, specialized cognitive load only affects processing of the first distractor but not subsequent distractors. Such a characteristic may be attributed to switch of attention from visually similar information (face) to irrelevant one (color dot).

In the second color judgment task, a pattern of reaction time seems to be consistent with that under general cognitive load (i.e., higher cognitive load increased distractor interference), although inconsistency across three experiments must be noted. In Experiment 1, reaction time for the second target judgment task was slower in the 3-load condition than the noload condition, indicating that distractor interference increased under high cognitive load. Similarly, in Experiment 2, reaction time for the second task was slower in the 3-load condition than the 1-load condition, regardless of load type. Another finding was that reaction time for the second targetjudgment task was slower in the face conditions than the scene conditions across load. Previous neuroimaging studies have shown that distractors whose category is congruent with cognitive load activated the prefrontal cortex (Dolcos et al., 2007; Jha et al., 2004; Minamoto et al., 2010), which is thought to provide domain-general cognitive resources (de Fockert et al., 2001). In that sense, the result may be also consistent with a 
characteristic of general cognitive load. In Experiment 3, reaction time for the second target judgment task did not differ across load, which was inconsistent with Experiments 1 and 2. However, reaction time in the high WMC group was faster than that in the low WMC group. Given that high WMC individuals efficiently assign cognitive resource in performing a cognitive task (Just \& Carpenter, 1992), the result could reflect difference in usage of domain-general cognitive resource. Those results in the second target judgment task seem to be in agreement with a characteristic of general cognitive load; however, it remains unclear why such pattern was obtained after exposure of the first distractor. This requires further investigation.

\section{Conclusions}

The present study demonstrated that the amount of specialized cognitive load is a critical factor in the emergence of memorydriven attentional capture effects (greater distractor interference). When the specialized load is low, distractors with similar visual characteristics of the load capture attention. When the specialized load is high, such attentional capture was no longer obtained because of depletion of attentional resource to process the specialized items. We also provided evidence that memory-driven attentional capture is an automatic attentional process as both high and low WMC individuals had their attention captured by distractors whose visual characteristics were similar to those in visual working memory. Furthermore, the multiple distraction procedure showed that the effect of the specialized cognitive load is restricted to the first contact of the visually similar distractors, and this effect vanished by repetitive exposure to the distractor, and the reaction time pattern seems to be consistent with that under general cognitive load. Future studies are required to investigate why the load effect was restricted to the first distractor items, considering an interactive mechanism between visual working memory, visual selective attention and executive attentional control.

Acknowledgments This study was supported by the Japan Society for the Promotion of Science, grant numbers 20-5480 and 23800034 to TM, and 22220003 to NO.

\section{References}

Alvarez, G. A., \& Cavanagh, P. (2004). The capacity of visual short-term memory is set both by visual information load and by number of objects. Psychological Science, 15(2), 106-111.

Cheung, O. S., \& Gauthier, I. (2010). Selective interference on the holistic processing of faces in working memory. Journal of Experimental Psychology: Human Perception and Performance, 36(2), 448-461. doi:10.1037/a0016471
Conway, A. R., Cowan, N., \& Bunting, M. F. (2001). The cocktail party phenomenon revisited: The importance of working memory capacity. Psychonomic Bulletin \& Review, 8(2), 331-335.

Daneman, M., \& Carpenter, P. A. (1980). Individual differences in working memory and reading. Journal of Verbal Learning and Verbal Behavior, 19, 450-466.

de Fockert, J. W., Rees, G., Frith, C. D., \& Lavie, N. (2001). The role of working memory in visual selective attention. Science, 291(5509), 1803-1806. doi:10.1126/science.1056496291/5509/1803

Dolcos, F., Miller, B., Kragel, P., Jha, A., \& McCarthy, G. (2007). Regional brain differences in the effect of distraction during the delay interval of a working memory task. Brain Research, 1152, 171-181.

Eriksen, B. A., \& Eriksen, C. W. (1974). Effects of noise letters upon the identification of a target letter in a non-search task. Perception \& Psychophysics, 16, 143-149.

Gao, W., Cao, B., Shan, S., Chen, X., Zhou, D., Zhang, X., \& Zhao, D. (2008). The CAS-PEAL large-scale Chinese face database and baseline evaluations. IEEE Transactions on System Man, and Cybernetics (Part A), 38(1), 149-161.

Horry, R., Wright, D. B., \& Tredoux, C. G. (2010). Recognition and context memory for faces from own and other ethnic groups: A remember-know investigation. Memory and Cognition, 38(2), 134-141. doi:10.3758/MC.38.2.134

Jha, A. P., Fabian, S. A., \& Aguirre, G. K. (2004). The role of prefrontal cortex in resolving distractor interference. Cognitive, Affective, \& Behavioral Neuroscience, 4(4), 517-527.

Just, M. A., \& Carpenter, P. A. (1992). A capacity theory of comprehension: Individual differences in working memory. Psychological Review, 99(1), 122-149.

Kane, M. J., \& Engle, R. W. (2002). The role of prefrontal cortex in working-memory capacity, executive attention, and general fluid intelligence: An individual-differences perspective. Psychonomic Bulletin and Review, 9(4), 637-671.

Kane, M. J., \& Engle, R. W. (2003). Working-memory capacity and the control of attention: The contributions of goal neglect, response competition, and task set to Stroop interference. Journal of Experimental Psychology: General, 132(1), 47-70.

Kim, S. Y., Kim, M. S., \& Chun, M. M. (2005). Concurrent working memory load can reduce distraction. Proceedings of the National Academy of Sciences of the United States of America, 102(45), 16524-16529. doi:10.1073/pnas.0505454102

Lang, P. J., Bradley, M. M., \& Cuthbert, B. N. (2008). International affective picture system (IAPS): Affective ratings of pictures and instruction manual. Technical Report A-8. Gainesville, FL: University of Florida.

Lavie, N., Hirst, A., de Fockert, J. W., \& Viding, E. (2004). Load theory of selective attention and cognitive control. Journal of Experimental Psychology: General, 133(3), 339-354. doi:10.1037/0096-3445. 133.3.339 2004-17825-003

Malpass, R. S., \& Kravitz, J. (1969). Recognition for faces of own and other face. Journal of Personality and Social Psychology, 13(4), 330-334.

McNab, F., \& Klingberg, T. (2008). Prefrontal cortex and basal ganglia control access to working memory. Nature Neuroscience, 11(1), $103-107$.

Minamoto, T., Osaka, M., Engle, R. W., \& Osaka, N. (2012). Incidental encoding of goal irrelevant information is associated with insufficient engagement of the dorsal frontal cortex and the inferior parietal cortex. Brain Research, 1429, 82-97. doi:10.1016/j.brainres.2011. 10.034

Minamoto, T., Osaka, M., \& Osaka, N. (2010). Individual differences in working memory capacity and distractor processing: Possible contribution of top-down inhibitory control. Brain Research, 1335, 6373. doi:10.1016/j.brainres.2010.03.088 
Olivers, C. N., Meijer, F., \& Theeuwes, J. (2006). Feature-based memorydriven attentional capture: Visual working memory content affects visual attention. Journal of Experimental Psychology: Human Perception and Performance, 32(5), 1243-1265. doi:10.1037/ 0096-1523.32.5.1243

Park, S., Kim, M. S., \& Chun, M. M. (2007). Concurrent working memory load can facilitate selective attention: Evidence for specialized load. Journal of Experimental Psychology: Human Perception and Performance, 33(5), 1062-1075.

Robinson, A., Manzi, A., \& Triesch, J. (2008). Object perception is selectively slowed by a visually similar working memory load. Journal of Vision, 8(16), 1-13. doi:10.1167/8.16.7/8/16/7/

Sobel, K. V., Gerrie, M. P., Poole, B. J., \& Kane, M. J. (2007). Individual differences in working memory capacity and visual search: The roles of top-down and bottom-up processing. Psychonomic Bulletin and Review, 14(5), 840-845.

Soto, D., Hodsoll, J., Rotshtein, P., \& Humphreys, G. W. (2008). Automatic guidance of attention from working memory. Trends in Cognitive Sciences, 12(9), 342-348. doi:10.1016/j.tics.2008.05.007

Soto, D., \& Humphreys, G. W. (2007). Automatic guidance of visual attention from verbal working memory. Journal of Experimental Psychology: Human Perception and Performance, 33(3), 730737. doi:10.1037/0096-1523.33.3.730

Soto, D., \& Humphreys, G. W. (2008). Stressing the mind: The effect of cognitive load and articulatory suppression on attentional guidance from working memory. Perception \& Psychophysics, 70(5), 924-934.

Theeuwes, J., \& Burger, R. (1998). Attentional control during visual search: The effect of irrelevant singletons. Journal of Experimental
Psychology: Human Perception and Performance, 24(5), 1342-1353.

Theeuwes, J., \& Van der Stigchel, S. (2006). Faces capture attention: Evidence from inhibition of return. Visual Cognition, 13(6), 657-665.

Turner, M. L., \& Engle, R. W. (1989). Is working memory capacity task dependent? Journal of Memory and Language, 28, 127154.

Unsworth, N., \& Engle, R. W. (2007). The nature of individual differences in working memory capacity: Active maintenance in primary memory and controlled search from secondary memory. Psychological Review, 114(1), 104-132. doi:10.1037/0033-295X. 114.1.104

Unsworth, N., Heitz, R. P., Schrock, J. C., \& Engle, R. W. (2005). An automated version of the operation span task. Behavior Research Methods, 37(3), 498-505.

Unsworth, N., Redick, T. S., Heitz, R. P., Broadway, J. M., \& Engle, R. W. (2009). Complex working memory span tasks and higher-order cognition: A latent-variable analysis of the relationship between processing and storage. Memory, 17(6), 635-654. doi:10.1080/ 09658210902998047

Unsworth, N., Schrock, J. C., \& Engle, R. W. (2004). Working memory capacity and the antisaccade task: Individual differences in voluntary saccade control. Journal of Experimental Psychology: Learning, Memory, and Cognition, 30(6), 1302-1321.

Vogel, E. K., McCollough, A. W., \& Machizawa, M. G. (2005). Neural measures reveal individual differences in controlling access to working memory. Nature, 438(7067), 500-503. 